\title{
ESCALA DE ENSOÑACIONES SEXUALES: PRIMEROS DA- TOS PSICOMÉTRICOS EN MUESTRAS ESPAÑOLAS
}

\author{
M" Isabel Vledma \\ Pllar Gutiérrez \\ Virgillo Ortega \\ Juan Carlos Slerra \\ Universidad de Granada
}

\section{RESUMEN}

En este estudio se han examinado algunas de las propiedades psicométricas de la versión española de la Escala de Ensoñaciones Sexuales de Giambra y Singer. Una muestra de 200 participantes (86 hombres y 114 mujeres), con una edad media de 24,36 años, completaron la Escala de Ensoñaciones Sexuales (SDS), el Cuestionanio de Wilson de Fantasias Sexuales (SFQ), el Inventario de Deseo Sexual (SDI) y la Encuesta de Opinión Sexual (SOS). Los resultados del análisis factorial confirmatorio mostraron un ajuste insuficiente de los datos al modelo unidimensional de la SDS. El análisis factorial exploratorio proporcionó tres factores interpretables: Tendencia a ensoñaciones heterosexuales, Intensidad de las ensoñaciones sexuales y Ensoñaciones eróticas personalizadas. Además, las tres dimensiones resultaron distinguibles mediante análisis factorial confirmatorio si uno de los items de la escala era eliminado; estas dimensiones mostraron correlaciones adecuadas con las variables criterio y valores alfa de Cronbach entre 
0,71 y 0,82 . A continuación, la estructura trifactorial fue replicada en otra muestra $(N=135 ; 60$ hombres y 75 mujeres, con edad media de 30,36 años). Se discuten las implicaciones de nuestros resultados sobre las propiedades psicométricas de la versión española y la evaluación de las ensoñaciones sexuales.

Palabras clave: PROPIEDADES PSICOMÉTIICAS, ENSOÑACIONES SEXUALES, FANTASÍAS SEXUALES, DESEO SEXUAL, ACTITUDES SEXUALES.

\section{SUMMARY}

The current study has examined some psychometric properties of the Giambra and Singer Sexual Daydreaming Scale' Spanish version. A sample of 200 participants (86 men and 114 women) with a 24.36 year-old stocking completed the Sexual Daydreaming Scale (SDS), the Wilson Sex Fantasy Questionnaire (SFQ), the Sexual Desire Inventory (SDI), and the Sexual Opinion Survey (SOS). Results of confirmatory factor analysis showed a poor fit to the data for the SDS's one-factor model. Exploratory factor analysis provided three interpretable factors: Propensity for heterosexual daydreaming, Intensity of sexual daydreams, and Personalized erotic daydreams. Also, these three dimensions were distinguishable using CFA if one item was deleted; they showed adequate correlations with criterion variables and Cronbach's alpha values between 0.71 and 0.82 . Next, threefactor structure was replicated on another sample ( $N=135 ; 60$ men and 75 women with a 30.06 year-old stocking). Implications of our results conceming psychometric properties of the Spanish version and sexual daydreaming assessment are discussed.

Key wOrds: PSYCHOMETRIC PROPERTIES, SEXUAL DAYDREAMING, SEXUAL FANTASIES, SEXUAL DESIRE, SEXUAL ATTIUIDES.

\section{INTRODUCCIÓN}

En la sexualidad humana, las fantasías sexuales tienen un papel muy relevante, equiparándose sus efectos a los de la estimulación 
física, actuando ambos factores de forma interactiva (Kaplan, 1974; Zubeidat y Sierra, 2003). Diversos estudios han puesto de relieve la relación existente entre la experiencia de fantasías sexuales y el deseo sexual (Sierra, Zubeidat, Carretero-Dios y Reina, 2003; Smith, Becker, Byrne y Pryzbyla, 1993; Zubeidat, Ortega, Del Villar y Sierra, 2003). Aunque Freud y otros psicoanalistas mantenian que las fantasías sexuales son resultado de la insatisfacción y la frustración sexual, cada vez existe más evidencia que identifica las fantasías sexuales como un indicador de salud y satisfacción sexual (Zimmer, Borchardt y Fischle, 1983) y la ausencia de las mismas como un signo de disfunción (Nutter y Condron, 1983, 1985), constituyéndose en una herramienta útil en terapia sexual (Heiman, LoPiccolo y LoPiccolo, 1976; LoPiccolo y Friedman, 1988; Sierra y Buela-Casal, 2004; Zeiss, Rosen y Zeiss, 1977; Zimmer et al., 1983). Sin embargo, también debemos tener en cuenta que no siempre las fantasías sexuales se viven de forma placentera sino que, en ocasiones, la culpabilidad por experimentarlas puede llevar a una situación de insatisfacción (Renaud y Byers, 1999, 2001; Zubeidat, Ortega y Sierra, 2004).

Leitenberg y Henning (1995) definen las fantasías sexuales como cualquier imagen mental que adquiere significado erótico o sexual para el individuo. Según Wilson (1978), las fantasías sexuales pueden constituir una experiencia imaginaria o pueden construirse sobre algún hecho real y/o experiencia pasada; pueden ser pensamientos rápidos y breves o historias muy bien confeccionadas referentes a algún hecho sexual, ocurriendo de forma espontánea, incluso fuera de la actividad sexual, o de forma deliberada. Las fantasias sexuales pueden ser inducidas tanto por señales internas (pensamientos o sentimientos) como por señales físicas externas, aunque no es necesaria la presencia de estímulos físicos para que sean evocadas. Algunos investigadores (Sierra, Ortega, Martín-Ortiz y Vera-Villarroel, 2004; Sierra, Ortega y Zubeidat, en prensa; Sierra, Vera-Villarroel y MartínezOrtiz, 2002; Wilson, 1988) han dirigido sus estudios a clasificar las fantasías sexuales, explorando cuales son más frecuentes o cuáles difieren entre mujeres y hombres. Con independencia del tipo de fantasías sexuales que puedan desarrollar los individuos, consideramos relevante conocer -incluso como paso previo a la evaluación del tipo de fantasías sexuales- la propensión que una 
persona tiene para fantasear o tener ensoñaciones sexuales; el concepto que mejor refleja esta propensión es el de ensoñación sexual.

A pesar de que en Psicología no existe una definición consensuada de ensoñación, Giambra (2000) la concibe como un pensamiento o imagen que ocurre de forma espontánea (no elicitada por recuerdos 0 estímulos perceptivos) y no realizable de forma inmediata. Asi, la ensoñación sexual puede ser diferente a la fantasía sexual, pues esta última puede ocurrir como ensoñación o de forma deliberada como pensamiento conscientemente manipulado (Purifoy, Grodsky y Giambra, 1992); es decir, la fantasía sexual englobaría a la ensoñación sexual. Otra diferencia entre ensoñaciones y fantasías sexuales estaría en sus contenidos; mientras el contenido de las primeras podría ser fantástico o real, el de las fantasías sexuales sería siempre fantástico o irreal (Purifoy et al., 1992). Por tanto, podríamos considerar la ensoñación sexual como un indicador de la propensión para desarrollar imágenes o pensamientos de naturaleza sexual, convirtiéndose su evaluación, a nuestro modo de entender, en el primer paso del estudio de las fantasías sexuales, en tanto un individuo que no experimente ensoñaciones sexuales difícilmente podrá desarrollar fantasías sexuales. Giambra y Martin (1977) informan de correlaciones positivas entre frecuencia de ensoñaciones sexuales y número de compañeros sexuales y frecuencia coital en los primeros dos años de matrimonio; Giambra (1983) asocia estas ensoñaciones con un mayor interés sexual en mujeres de mediana edad. Purifoy et al. (1992) encontraron en una muestra de mujeres que las ensoñaciones sexuales estaban asociadas a un mayor interés y actividad sexual y a actitudes positivas hacia el sexo.

Dado que las fantasias sexuales son un aspecto relevante a valorar de la sexualidad humana, se pone de manifiesto la necesidad de disponer de instrumentos con adecuadas garantias psicométricas que permitan evaluar la propensión a fantasear de las personas, las actitudes asociadas a las fantasías sexuales y los contenidos de las mismas. La mayoría de estos instrumentos se ha elaborado originalmente en países anglosajones, por lo que su empleo en países de habla hispana requiere un proceso de adaptación para que cumplan con las garantías psicométricas necesarias. En España se han realizado estudios psicométricos de algunos instrumentos para evaluar 
las actitudes hacla las fantasías sexuales y los contenidos de las mismas. El Hurtbert Index of Sexual Fantasy (Hurlbert y Apt, 1993) permite evaluar la actitud de un individuo hacia las fantasfas sexuales. Desvarieux, Salamanca, Ortega y Sierra (2005) llevaron a cabo la validación de la versión en castellano de esta escala; su versión reducida de 10 ítems obtuvo una elevada consistencia interna (alfa de Cronbach $=0,85$ ) y soporte para las hipótesis de validación establecidas con las fantasías sexuales y el deseo sexual. Con respecto a la evaluación del tipo de fantasías sexuales, el instrumento más utilizado ha sido el Cuestionario de Fantasías Sexuales (Wilson, 1988). Sierra et al. (2004) informan de coeficientes alfa de Cronbach entre 0,66 y 0,79 para las diferentes subescalas que componen la prueba (fantasias sexuales exploratorias, íntimas, impersonales y sadomasoquistas) así como de una estructura factorial obtenida mediante análisis de componentes principales bastante similar a la propuesta original de sus autores; en el estudio de Carvajal, Sebastián, Cornide, Delgado, Castellote y Blanco (1990) se mostró igualmente un apoyo parcial a la estructura dimensional del cuestionario mediante análisis factorial exploratorio. Sierra et al. (en prensa) plantean una versión reducida del instrumento que mejora considerablemente su nivel de ajuste al modelo térico original de cuatro factores correlacionados mediante análisis factorial confirmatorio, tanto en hombres como en mujeres. En la evaluación de la propensión a experimentar ensoñaciones sexuales destaca la Sexual Daydreaming Scale (SDS) (Giambra y Singer, 1998). Este instrumento constituye una subescala del Imaginal Processes Inventory (IPI) (Singer y Antrobus, 1963, 1972), que evalúa varias dimensiones de las ensoñaciones y su relación con procesos mentales como la atención, distractibilidad o curiosidad (Giambra, 1979).

Dada la ausencia de estudios psicométricos en muestras españolas, el objetivo de este estudio instrumental (Carretero-Dios y Pérez, en prensa; Montero y León, 2005) es analizar algunas de las características psicométricas de la versión española del Sexual Daydreaming Scale (SDS). Con esta finalidad, se analizó la estructura factorial de la escala, su consistencia intema y las correlaciones con algunos criterios como diferentes tipos de fantasias sexuales, deseo sexual y actitudes sexuales. Igualmente, se evaluaron las diferencias 
en el instrumento entre mujeres y hombres, por una parte, y entre individuos con y sin pareja, por otra. Por último, se trató de replicar la estructura factorial del instrumento en una muestra distinta.

\section{MÉTODO}

\section{Muestras}

Se han utilizado dos muestras diferentes seleccionadas mediante procedimiento no aleatorio. La muestra 1 estaba formada por 200 participantes (86 hombres y 114 mujeres), cuya edad oscilaba entre 18 y 36 años (media $=24,36$; desviación típica $=4,44$ ); todos ellos eran estudiantes universitarios, de entre los cuales 110 tenían pareja en el momento de la evaluación desde hacla al menos 3 meses y 90 no cumplían esta condición La muestra 2 la componían 135 estudiantes universitarios ( 60 hombres y 75 mujeres) con edades comprendidas entre 19 y 48 años (media = 30,06; desviación típica $=7,77$ ), de los cuales 50 tenian pareja y 85 no. En ambas muestras todos los participantes indicaron una orientación heterosexual.

\section{Instrumentos}

- Sexual Daydreaming Scale (SDS) (Giambra y Singer, 1998). Permite evaluar las ensoñaciones de tipo sexual o naturaleza erótica. Está formada por 12 ítems que se contestan en una escala tipo Likert con cinco opciones de respuesta (desde $1=$ totalmente falso en mí hasta 5 = muy cierto en $\mathrm{m}$ ), oscilando la puntuación total entre 12 (ausencia de ensoñaciones sexuales) y 60 (elevada presencia de ensoñaciones sexuales). Sus autores informan de una consistencia interna cuyos valores alfa de Cronbach oscilan entre 0,87 y 0,93 (Giambra, 1977, 1979; Singer y Antrobus, 1972); las puntuaciones de la SDS correlacionan significativamente con la edad (Giambra, 1979), el número de parejas sexuales y número de coitos (Giambra y Martín, 1977) y con los síntomas de la menopausia, frecuencia masturbatoria e interés sexual por la pareja (Giambra, 1983). En el Anexo 1 se incluye la versión española de este instrumento, en la cual hemos optado por utilizar el término "fantasía" (fantasy) en lugar de "ensoñación" 
(daydreaming) -pese a tratarse de conceptos diferentes, tal y como queda reflejado en la introducción de este trabajo-. El motivo que justifica esta decisión es la escasa utilización del segundo término en el lenguaje cotidiano; en la fase de traducción pudimos apreciar que los individuos con conocimiento lego en el tema tienden a equiparar ambos conceptos.

- Sex Fantasy Questionnaire (SFQ) (Wilson, 1988). Incluye 32 items distribuidos en cuatro subescalas: fantasias sexuales exploratorias, intimas, impersonales y sadomasoquistas. Sierra et al. (2004) analizan su validez y fiabilidad en una muestra española, encontrándose un apoyo parcial a la estructura dimensional planteada por los autores e informando de valores alfa de Cronbach entre 0,66 y 0,79 para las diferentes subescalas, asi como de una adecuada validez convergente con la subescala de Comportamiento sexual deseado del Inventario de Interacción Sexual de LoPiccolo y Steger (1974). En un estudio más reciente, Sierra et al. (en prensa) utilizan una versión reducida de 24 ítems que mejora el ajuste al modelo de cuatro factores oblicuos y establece correlaciones significativas con el deseo sexual y las actitudes sexuales; los valores de consistencia interna de las cuatro subescalas oscilan entre 0,73 y 0,85 . En el presente estudio se utilizó esta versión reducida.

- Sexual Desire Inventory (SDI) (Spector, Carey y Steinberg, 1996). Está integrado por 13 ítems distribuidos en dos subescalas: deseo sexual diádico y deseo sexual solitario. Sus autores informan de valores elevados de consistencia interna para ambas subescalas $(0,85$ y 0,96 , respectivamente). En la adaptación española, Ortega, Zubeidat y Sierra (2005) replican la estructura factorial oniginal y obtienen valores alfa de Cronbach de 0,87 para la escala de deseo sexual diádico y 0,88 para la de deseo sexual solitario; las puntuaciones de las dos subescalas correlacionan significativamente con las fantasías sexuales y la erotofilia.

- Sexual Opinion Survey (SOS) (Fisher, Byme, White y Kelly, 1988). Consta de 21 ítems a través de los cuales evalúa la respuesta afectiva del individuo ante diferentes estímulos sexuales (dimensión erotofobiaerotofilia). Carpintero y Fuertes (1994) informan de una consistencia interna global de 0,86 para la adaptación española. Por su parte, Lameiras y Failde (1998) señalan una estructura multidimensional con 
cuatro factores: erotofilia, erotofobia, homofobia y sexo no convencional. En el presente estudio se utilizaron los ítems que corresponden a las dimensiones erotofilia, homofobia y sexo no convencional.

\section{Procedimiento}

En la traducción al castellano de la Sexual Daydreaming Scale (SDS) se empleó el procedimiento de traducción directa o hacia delante (Hambleton, 1993, 1996), prestando especial atención a los sesgos de carácter idiomático y metafórico, tal como recomienda Brislin (1986). La traducción fue revisada por dos estudiantes de postgrado en Psicología bilingïes. A continuación dos profesores expertos en sexualidad humana revisaron la redacción de los ítems. Una vez superadas estas fases se procedió a la administración de la versión definitiva de la Escala de Ensoñaciones Sexuales junto al resto de autoinformes de forma colectiva en la primera muestra. La aplicación fue realizada por dos evaluadores en una sola sesión dando a todos los participantes las mismas instrucciones. Las respuestas eran completamente anónimas. Con la segunda muestra se siguió exactamente el mismo procedimiento, si bien en este caso los participantes solo recibían la Escala de Ensoñaciones Sexuales.

\section{RESULTADOS}

\section{Análisis estadísticos}

Se realizó un análisis factorial confirmatorio (AFC) con LISREL 8.54 para evaluar el ajuste a los datos de los modelos propuestos, incluyendo como línea de base el modelo nulo que hipotetiza la independencia entre todas las variables observadas. Los indices utilizados en la evaluación de los modelos fueron el ratio ji-cuadrado por grados de libertad $(\div 2 / d f)$, el índice de Tucker-Lewis (TLI) (Tucker y Lewis, 1973), el índice no centralizado relativo (RNI) (McDonald y Marsh, 1990) y el error de aproximación cuadrático medio (RMSEA) (Browne y Cudeck, 1989; Steiger, 1990). Para el ratio $\div 2 / d$, valores entre 1 y 3 indican un buen ajuste (Carmines y Mclver, 1981), pudiendo ampliarse el umbral superior hasta un límite más laxo de 5 (Jöreskog, 
1970). TLI y RNI difieren principalmente en que el T'LI, al considerar los grados de libertad de los modelos, elimina el problema del sobreajuste como consecuencia de añadir más parámetros a medida que aumenta la complejidad del modelo (McDonald y Marsh, 1990). Consideramos susceptibles de mejora los valores por debajo de 0,95, si bien la mayor utilidad de los índices reside en su capacidad para comparar el ajuste a los datos entre modelos alternativos. EI RMSEA ha sido considerado como el mejor indicador del ajuste global del modelo (Marsh, Balla y Hau, 1996). El nivel recomendado para buen ajuste del modelo es RMSEA inferior a 0,05. Browne y Cudeck (1993) consideran que valores en el rango de 0,05 a 0,08 indican un ajuste aceptable o moderado, mientras que valores por encima de 0,10 indican un ajuste deficiente. Siguiendo a Du Toint y Du Toint (2001), partimos de la matriz de correlaciones policórica y la matriz de covarianzas asintótica para realizar todas las estimaciones, más adecuadas al trabajar con variables ordinales. Se utilizó el método de estimación de mínimos cuadrados ponderados (WLS).

Se recurrió al análisis factorial exploratorio (AFE) con SPSS 12.0 para indagar la estructura subyacente a los ítems de la escala sin plantear hipótesis al respecto. En este caso, empleamos análisis factorial de cornponentes principales, sin prefijar el número de factores y aplicando rotación ortogonal Varimax con Kaiser. Previamente, se constató la adecuación de los datos a la técnica mediante el test de adecuación de muestreo de Kaiser-Meyer-Olkin (KMO) y la prueba de esfericidad de Bartlett. Para determinar el número de factores, se utilizó la regla Kaiser-Guttman (Guttman, 1954; Kaiser, 1960, 1970), que retiene componentes con valores Eigen iguales o superiores a 1. Se establecieron los siguientes criterios para que un elemento formara parte de un factor: a) alcanzar una carga factorial igual o superior a 0,40 en la matriz de componentes rotados; b) en caso de existir una saturación de esa cuantía en más de un factor, ha de existir una diferencia en saturación mínima de 0,15 en valor absoluto para ser seleccionado en un solo factor; c) han de elegirse factores que contengan al menos dos elementos. La consistencia interna entre grupos de ítem fue estimada mediante alfa de Cronbach; también se calcularon correlaciones y estadísticos descriptivos. Las diferencias de medias entre factores para las variables sexo y pareja fueron contrastadas mediante la prueba $t$ de Student. 


\section{Estructura factorial: análisis confirmatorio I}

En la Tabla 1 se muestran los resultados del análisis factorial confirmatorio. El modelo unifactorial obtuvo un ajuste insatisfactorio. $A$ pesar de que el ratio ji-cuadrado por grados de libertad se encuentra dentro del rango permitido $(\div / d f=2,97)$, los índices TLI y RNI resultaron inferiores a 0,95 y el error de aproximación cuadrático medio se situó por encima de lo que se considera un ajuste moderado o aceptable (RMSEA $=0,099$ ). Dado que el ftem 2 presentaba el error más elevado y saturación más baja de todos los elementos $(0,77$ y 0,48 , respectivamente), se procedió a comprobar el ajuste del modelo unifactorial excluyendo el ítem del conjunto. En este caso, el modelo de un solo factor con los 11 ítems restantes tampoco consiguió un ajuste adecuado a los datos, obteniendo el mismo ratio y error de aproximación cuadrático medio $(\div 2 / d f=2,97 ; \operatorname{RMSEA}=0,099)$.

TABLA 1. Estadísticos de bondad de ajuste para los modelos propuestos sobre la Escala de Ensoñaciones Sexuales.

\begin{tabular}{lccccc}
\hline Modelos & $\chi^{2 \mathrm{a}}$ & $d f$ & TLI & RNI & RMSEA \\
\hline Modelo Nulo (12 ítems) & 1535,32 & 66 & $\mathrm{NA}^{\mathrm{b}}$ & $\mathrm{NA}^{\mathrm{b}}$ & 0,334 \\
Modelo de 1 factor (12 items) & 160,30 & 54 & 0,91 & 0,93 & 0,099 \\
Modelo de 1 factor (11 items) & 130,53 & 44 & 0,92 & 0,93 & 0,099 \\
\hline
\end{tabular}

a Los valores ji-cuadrado son significativos $(p<0,001) ; \mathrm{b} A=$ no aplicable.

\section{Estructura factorlal: análisis exploratorio}

Los resultados del test de Kaiser-Meyer-Olkin $(\mathrm{KMO}=0,86)$ y la prueba de esfericidad de Bartlett ( $\div=862,79 ; p<0,001$ para $66 \mathrm{gl}$ ) indicaron que los datos son adecuados para el uso de la técnica. Se aislaron tres factores que explicaron el $60,88 \%$ de la varianza total. En la Tabla 2 puede observarse la solución factorial obtenida mediante normalización de Kaiser, junto a la media y desviación típica para cada ítem.

El factor 1 (F1) explicó el $41,47 \%$ de la varianza total, con un valor propio de 6,25; este factor apareció constituido por los ítems $4,8,3,11$ y 6 ; todos ellos son elementos vinculados a la propensión a la 
TABLA 2. Matriz de componentes rotados, media, desviación típica y varianza total explicada en la Escala de Ensoñaciones Sexuales $(N=200)$

\begin{tabular}{|c|c|c|c|c|c|}
\hline Item & Media & DT & F1 & $\bar{F} 2$ & F3 \\
\hline 4 & 2,05 & 1,12 & 0,75 & & \\
\hline 8 & 2,60 & 1,13 & 0,72 & & \\
\hline 3 & 2,06 & $\cdot 1,03$ & 0,71 & & \\
\hline 11 & 2,55 & 1,18 & 0,69 & & \\
\hline 6 & 2,01 & 1,04 & 0,60 & & \\
\hline 1 & 2,82 & 1,04 & & 0,79 & \\
\hline 5 & 2,93 & 1,03 & & 0,71 & \\
\hline 12 & 3,00 & 1,26 & 0,41 & 0,69 & \\
\hline 2 & 2,94 & 1,03 & & 0,53 & \\
\hline 10 & 2,48 & 1,22 & 0,47 & & 0,88 \\
\hline 9 & 2,56 & 1,23 & & & 0,66 \\
\hline 7 & 2,53 & 1,12 & & & 0,63 \\
\hline & \multicolumn{2}{|c|}{ Eigenvalue } & 6,25 & 1,75 & 1,27 \\
\hline & \multicolumn{2}{|c|}{$\%$ Varianza } & 41,47 & 10,96 & 8,45 \\
\hline
\end{tabular}

ensoñación y los dos items que alcanzan mayor saturación en el componente se refieren de forma explícita a ensoñaciones con personas del sexo opuesto. Por ello, nos inclinamos a denominar a este primer componente como Tendencia a ensoñaciones heterosexuales. El factor 2 (F2) explicó el 10,96\% de la varianza total, con un valor propio de 1,75, y estuvo formado por los ítems $1,5,12$ y 2; los elementos que forman parte de este componente se relacionan con la viveza o grado de nitidez de las ensoñaciones sexuales y el nivel de activación que éstas provocan en el individuo, lo que nos condujo a designar este segundo factor como Intensidad de las ensoñaciones sexuales. Únicamente el ítem con menor saturación en este componente (ítem 2) alude al tipo de contenido recogido en la fantasía ("Yo me imagino físicamente atractivo para las personas del sexo opuesto"). El tercer factor (F3) explicó el $8,45 \%$ de la varianza total, con un valor propio de 1,27. Este componente englobó los items 10, 9 y 7 , de entre los cuales dos se refieren a tener ensoñaciones con alguien a quién se aprecia o admira, mientras que el último se relaciona con la propensión a pensamientos amorosos; el factor ha sido etiquetado como Ensoñaciones eróticas personalizadas. 


\section{Validez factorial: anállsis confirmatorio II}

En la Tabla 3 se muestran los estadísticos de bondad de ajuste para el modelo de tres factores relacionados hipotetizado sobre los 12 items que representaron la Tendencia a ensoñaciones heterosexuales (F1), Intensidad de las ensoñaciones (F2) y Ensoñaciones eróticas personalizadas (F3). El modelo de tres factores oblicuos no consiguió un ajuste adecuado a los datos; a pesar del adecuado ratio ji-cuadrado por grados de libertad $(\div 2 / d f=2,48)$, el RMSEA supera el límite de 0,080 . Al igual que en la solución unifactorial, el ítem 2 presentó elevado error y baja carga factorial $(0,73$ y 0,52 , respectivamente), por lo que probamos nuevamente el ajuste de los datos al modelo trifactorial excluyendo dicho ítem. De esta forma, los índices TLI y RNI obtuvieron valores adecuados $(0,95$ y 0,96 , respectivamente), al igual que el ratio $\div 2 / d f=2,10$; el RMSEA $=0,074$ indicó un ajuste aceptable.

TABLA 3. Estadísticos de bondad de ajuste para el modelo propuesto sobre tres componentes obtenidos en AFE

\begin{tabular}{l|c|c|c|c|c}
\hline Modelos & $\chi^{2 \pi}$ & $d f$ & TLI & RNI & RMSEA \\
\hline Modelo Nulo (11 items) & 1335,63 & 55 & NA $^{6}$ & NA $^{6}$ & 0,342 \\
Modelo de 3 factores oblicuos (12 items) & 126,68 & 51 & 0,93 & 0,95 & 0,086 \\
Modelo de 3 factores oblicuos (11 items) & 86,18 & 41 & 0,95 & 0,96 & 0,074 \\
\hline
\end{tabular}

aLos valores ji-cuadrado son significativos $(p<0,001) ;$ NA $=$ no aplicable.

\section{Consistencia Interna y correlaclones}

En la Tabla 4 se recogen las correlaciones entre los tres factores que han sido señalados, así como el número de ítems y consistencia interna (alfa de Cronbach) en cada uno de ellos, oscilando entre 0,71 de Ensoñaciones eróticas personalizadas y 0,82 de Tendencia a ensoñaciones heterosexuales.

En la Tabla 5 se incluyen las correlaciones entre cada uno de los factores de la Escala de Ensoñaciones Sexuales y diferentes variables criterio: tipos de fantasias sexuales (exploratorias, íntimas, impersonales y sadomasoquistas), deseo sexual (diádico y solitario) y actitudes sexuales (erotofilia, homofobia y sexo no convencional). Tal como se puede apreciar, las correlaciones positivas entre los tres factores y los tipos de fantasias sexuales resultaron todas significativas 
oscilando entre 0,28 (Ensoñaciones eróticas personalizadas con fantasías impersonales) y 0,51 (Tendencia a ensoñaciones heterosexuales con fantasías exploratorias). Por otra parte, las correlaciones positivas entre los tres factores de la escala de ensoñaciones y el deseo sexual (diádico y solitario) también resultaron significativas. Las correlaciones con las actitudes sexuales fueron más bajas; asi, resultaron significativas las correlaciones positivas de erotofilia con Tendencia a ensoñaciones heterosexuales $(r=0,14 ; p<$ $0,05)$ e Intensidad de las ensonaciones $(r=0,18 ; p<0,05)$, y de sexo no convencional con los tres factores de la escala de ensoñaciones; por último, se encontró una correlación negativa entre homofobia y Tendencia a ensoñaciones heterosexuales $(r=-0,18 ; p<0,01)$.

TABLA 4. Consistencia intema y correlaciones entre los tres factores oblicuos con 11 ítems

\begin{tabular}{lccc}
\cline { 2 - 4 } & \multicolumn{1}{c}{3} & 3 \\
\hline 1. Tendencia a ensoñaciones heterosexuales & - & & \\
2. Intensidad de las ensoñaciones & $0,55^{* * *}$ & - & \\
3. Ensorlaciones eróticas personalizadas & $0,59^{* * *}$ & $0,40^{* * *}$ & - \\
Número de ftems & 5 & 3 & 3 \\
Alfa & 0,82 & 0,74 & 0,71 \\
\hline
\end{tabular}

"**p<0,01;"*p<0,01; "p<0,05

TABLA 5. Consistencia intema y correlaciones de las variables criterio con los tres factores oblicuos (11 ftems)

\begin{tabular}{llll}
\cline { 2 - 4 } & \multicolumn{1}{c}{$F 1$} & $F 2$ & $F 3$ \\
\hline Fantasías exploratorias & $0,51^{* * *}$ & $0,43^{* * *}$ & $0,31^{* * *}$ \\
Fantasías íntimas & $0,49^{* * *}$ & $0,47^{* * *}$ & $0,44^{* * *}$ \\
Fantasías impersonales & $0,39 * * *$ & $0,33^{* * *}$ & $0,28^{* * *}$ \\
Fantasías sadomasoquistas & $0,38^{* * *}$ & $0,33^{* * *}$ & $0,42^{* * *}$ \\
Deseo sexual diádico & $0,52^{* * *}$ & $0,48^{* * *}$ & $0,52^{* * *}$ \\
Deseo sexual solitario & $0,46^{* * *}$ & $0,40^{* * *}$ & $0,23^{* *}$ \\
Erotofilia & $0,14^{*}$ & $0,18^{*}$ & 0,10 \\
Homofobia & $-0,18^{* *}$ & $-0,06$ & $-0,10$ \\
Sexo no convencional & $0,35^{* * *}$ & $0,27^{* * *}$ & $0,25^{* * *}$ \\
\hline
\end{tabular}

***p<0,01;"*p<0,01;"p<0,05 
Dlferencias entre hombres y mujeres, y entre Indlviduos con pareja y sin pareja

Se exploraron las diferencias en los tres factores entre hombres y mujeres, por una parte, y entre sujetos que tenían pareja y los que no la tenían, por otra. En ambos casos se utilizó la prueba $t$ de Student para muestras independientes. En la Tabla 6 se recogen las diferencias de medias entre el grupo de hombres $(n=86)$ y el de mujeres $(n=$ 114), manifestándose en los hombres una tendencia significativamente mayor a las ensoñaciones heterosexuales, así como una intensidad de las ensoñaciones sexuales significativamente más elevada. Por su parte, en la Tabla 7 se indican las diferencias entre aquellos individuos que tienen pareja $(n=110)$ y los que no tienen $(n=90)$; el grupo $\sin$ pareja presentó una tendencia a las ensoñaciones heterosexuales y ensoñaciones eróticas personalizadas significativamente mayor.

TABLA 6. Diferencias de medias en los tres factores de ensoñaciones sexuales entre hombres y mujeres

\begin{tabular}{llcccc} 
& Sexo & Media & DT & t & Sig. \\
\hline \multirow{2}{*}{ Tendencia ensoñaciones heterosexuales } & Hombre & 12,67 & 4,70 & 4,16 & 0,000 \\
& Mujer & 10,18 & 3,43 & & \\
Intensidad de las ensoñaciones sexuales & Hombre & 9,36 & 2,67 & & \\
& Mujer & 8,29 & 2,65 & 2,82 & 0,005 \\
Ensoñaciones eróticas personalizadas & Hombre & 7,60 & 2,89 & & \\
& Mujer & 7,52 & 2,82 & 0,21 & 0,831 \\
\hline
\end{tabular}

TABLA 7. Diferencias de medias en los tres factores de ensoñaciones sexuales entre individuos con y sin pareja

\begin{tabular}{|c|c|c|c|c|c|}
\hline & Pareja & Media & DT & $\mathbf{t}$ & Sig. \\
\hline Tendencia ensoñaciones heterosexuales & $\begin{array}{l}\text { Si } \\
\text { No }\end{array}$ & $\begin{array}{l}10,43 \\
12.26\end{array}$ & $\begin{array}{l}3,63 \\
4,64\end{array}$ & $-3,05$ & 0,003 \\
\hline Intensidad de las ensonaciones sexuales & $\begin{array}{l}\text { Si } \\
\text { No }\end{array}$ & $\begin{array}{l}8,83 \\
8,66\end{array}$ & $\begin{array}{l}2,43 \\
3,02\end{array}$ & 0,44 & 0,663 \\
\hline Ensoñaciones eróticas personalizadas & $\begin{array}{l}\text { Si } \\
\text { No }\end{array}$ & $\begin{array}{l}7,05 \\
8,18 \\
\end{array}$ & $\begin{array}{l}2,77 \\
2,82\end{array}$ & $-2,85$ & 0,005 \\
\hline
\end{tabular}




\section{Validación cruzada de la estructura factorial}

Para evaluar la estabilidad de la solución de tres factores oblicuos con 11 ítems, se seleccionó una segunda muestra de 135 individuos. En este caso, dado el tamaño de la muestra se recurrió al análisis de componentes principales. Los resultados de la prueba de esfericidad

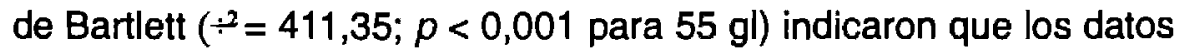
son adecuados para el uso de la técnica. En cuanto al test de KaiserMeyer-Olkin (KMO $=0,71)$, Kaiser (1974) considera los valores entre 0,70 y 0,80 como aceptables. Se aislaron tres factores que explicaron el $58,18 \%$ de la varianza total. En la Tabla 8 puede observarse la solución factorial obtenida mediante normalización de Kaiser, junto a la media y desviación típica para cada ítem.

TABLA 8. Matriz de componentes rotados, media, desviación típica y varianza total explicada en los la Escala de Ensoñaciones Sexuales $(N=135)$

\begin{tabular}{|c|c|c|c|c|c|}
\hline Item & Media & DT & $\overline{F I}$ & $\overline{F 2}$ & F3 \\
\hline 4 & 1,10 & 1,11 & 0,83 & & \\
\hline 6 & 0,95 & 1,01 & 0,73 & & \\
\hline 3 & 1,03 & 0,94 & 0,66 & & \\
\hline 8 & 1,55 & 1,17 & 0,52 & & 0,41 \\
\hline 12 & 1,94 & 1,23 & & 0,77 & \\
\hline 11 & 1,63 & 1,13 & & 0,63 & \\
\hline 1 & 1,98 & 1,09 & & 0,60 & \\
\hline 5 & 2,14 & 1,08 & & 0,55 & \\
\hline 10 & 1,57 & 1,26 & & & 0,88 \\
\hline 9 & 1,53 & 1,17 & 0,40 & & 0,74 \\
\hline 7 & 1,35 & 1,10 & & 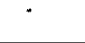 & 0,40 \\
\hline & \multicolumn{2}{|c|}{ Eigenvalue } & 4,95 & 1,64 & 1,44 \\
\hline & \multicolumn{2}{|c|}{$\%$ Varianza } & 35,89 & 11,87 & 10,43 \\
\hline
\end{tabular}

El factor 1 (F1) explicó el 35,89\% de-la varianza total, con un valor propio de 4,95; este factor apareció constituido por los ítems 4, 6, 3 y 8 , si bien el último de ellos aparece también como elemento de segundo orden en el factor 3 . El factor 2 (F2) explicó el $11,87 \%$ de la varianza total, con un valor propio de 1,64, y estuvo formado por los ítems 12 , 11,1 y 5 . El factor 3 (F3) explicó el $10,43 \%$ de la varianza total, con un valor propio de 1,44; este componente englobó los ítems 10, 9 y 7. 


\section{DISCUSIÓN}

Las fantasías sexuales constituyen un aspecto esencial de la sexualidad humana en la determinación del interés y la actividad sexual, siendo relevante su evaluación en terapia sexual, pues se pueden utilizar como técnica de reducción de los niveles de ansiedad (Sierra y BuelaCasal, 2004). A nuestro entender, antes de evaluar los tipos de fantasías sexuales que muestra un individuo, sería necesario determinar la predisposición que tiene para desarrollar ensoñaciones de tipo erótico. A pesar de que se han elaborado diferentes instrumentos de autoinforme para su evaluación, la mayoría de ellos es de origen anglosajón y muy pocos se centran en la predisposición para fantasear. Por ello, en el presente estudio nos planteamos realizar una estimación de la fiabilidad y validez de la versión española de la Escala de Ensoñaciones Sexuales (Sexual Daydreaming Scale, Giambra y Singer, 1998).

Dado que el análisis factorial confirmatorio realizado en primer lugar no sostuvo la presunta unidimensionalidad, a partir de análisis factorial exploratorio se propuso una estructura de tres factores oblicuos que explicaron un $60,88 \%$ de la varianza total, lo cual no concuerda con el planteamiento original de los autores de la escala (Giambra, 1979; Giambra y Martin, 1977; Giambra y Singer, 1998). El primer factor, denominado Tendencia a ensoñaciones heterosexuales, explicó el $41,47 \%$ de la varianza total e incluye elementos que evalúan la propensión a la ensoñación; el segundo factor, Intensidad de las ensoñaciones sexuales, explicó el $10,96 \%$ de la varianza total e incluye ítems relacionados con la viveza o grado de nitidez de las ensoñaciones sexuales y el nivel de activación que éstas elicitan en la persona; el tercer factor aislado, Ensoñaciones eróticas personalizadas, explicó el $8,45 \%$ de la varianza total, englobando la tendencia a tener ensoñaciones con alguien admirado o querido, asi como la propensión general de la persona a tener pensamientos de tipo amoroso. Todos los ítems que forman la escala se ajustan a este modelo de tres factores oblicuos, excepto el ítem 2 ("Yo me imagino físicamente atractivo para las personas del sexo opuesto"), el cual ha presentado un elevado error y una baja carga factorial.

Para evaluar la estabilidad de la solución de tres factores oblicuos con 11 items se llevó a cabo un segundo análisis factorial, utilizando 
para ello una segunda muestra. En este caso se volvieron a aislar de nuevo tres factores que explicaron el $58,19 \%$ de la varianza total, reproduciendo prácticamente la solución anterior. El único ítem que no ratifica la estructura obtenida en la primera muestra es el 11 ("Antes de dormirme, mis pensamientos ociosos se vuelven eróticos"), que aparecía en el primer factor y ahora se sitúa en el segundo, lo cual podría estar asociado a la activación que estos pensamientos eróticos provocan antes de dormir. Si bien los resultados de la segunda muestra replican casi perfectamente la propuesta trifactorial, sería interesante corroborar estos resultados en otros estudios con muestras diferentes.

Los tres factores aislados presentan relaciones significativas entre sí, oscilando éstas entre 0,40 y 0,59 , y su consistencia intema, calculada mediante el alfa de Cronbach, muestra valores adecuados, situándose entre 0,71 para el tercer factor y 0,82 para el primero. Dado que los estudios originales de la escala no señalan una estructura multidimensional, los valores de fiabilidad indicados por los autores hacen referencia a la escala total, situándose éstos entre 0,87 (Singer y Antrobus, 1972) y 0,93 (Giambra, 1977, 1979).

Se han encontrado correlaciones significativas entre los tres factores de la Escala de Ensoñaciones Sexuales y los cuatro tipos de fantasías sexuales (exploratorias, intimas, impersonales y sadomasoquistas), lo que evidencia que la propensión a fantasear manifestada por la presencia de ensoñaciones sexuales se asocia a una mayor frecuencia de distintos tipos de fantasias sexuales. También se encontraron correlaciones significativas de las tres subescalas con el deseo sexual, tanto diádico como solitario; estudios previos han puesto de manifiesto la asociación entre fantasias sexuales y deseo sexual, mostrando que éstas favorecen el deseo y la actividad sexual (Purifoy et al., 1992; Sierra et al., 2003; Smith et al., 1993; Zimmer et al., 1983; Zubeidat et al., 2003). Giambra y Martin (1977), utilizando una muestra de hombres, asociaron puntuaciones elevadas en la Escala de Ensoñaciones Sexuales con un mayor número de parejas sexuales, y un mayor número de coitos y actividades sexuales; asimismo, en mujeres se asoció la experiencia de ensoñaciones sexuales con la frecuencia masturbatoria y el interés por mantener relaciones sexuales con la pareja (Giambra, 1983). Con respecto a las actitudes sexuales, las correlaciones resultaron más leves, aunque 
en general indican una asociación significativa entre la experiencia de ensoñaciones sexuales y la disposición a actitudes sexuales positivas, lo que concuerda con otros estudios en los que las actitudes erotofílicas se asocian a un mayor número de fantasías sexuales (Brown y Hart, 1977; Fisher et al., 1988; Sierra et al., en prensa; Zurbriggen y Post, 2004); en esta misma línea, Purifoy et al. (1992) relacionaron las ensoñaciones sexuales con actitudes sexuales positivas. Por otra parte, existe evidencia de que los hombres suelen experimentar mayor cantidad de fantaslas sexuales que las mujeres (Ellis y Symons, 1990; García-Vega, Fernández-Garcla y Rico Fernández, 2005; Hicks y Leitenberg, 2001; Hsu, Kling, Kessler y Knapke, 1994; Leitenberg y Henning, 1995; Sierra et al., 2002; Wilson, 1997; Wilson y Lang, 1981). Los resultados encontrados en el presente estudio van en este mismo sentido; así, los hombres muestran una mayor tendencia a ensoñaciones heterosexuales y una mayor intensidad en su experiencia. Giambra (1979) señaló que los hombres muestran una puntuación media mayor que las mujeres en la escala de ensoñaciones sexuales. Por último, al comparar las puntuaciones de los tres factores de ensoñaciones sexuales obtenidas por los individuos con pareja y sin pareja, se ha puesto de manifiesto que estos últimos muestran una mayor tendencia a las ensoñaciones heterosexuales y a ensoñaciones eróticas personalizadas. Apenas existen trabajos que analicen el efecto de tener pareja o no sobre la experiencia de fantasías sexuales. En un reciente estudio, Sierra et al. (2004) aportan algunos resultados al respecto; así, los sujetos emparejados informaban en mayor medida de fantaslas relacionadas con el disfrute con un número limitado de parejas sexuales, mientras que los que no tenían pareja señalaban un mayor número de fantasías asociadas a la variedad sexual; futuras investigaciones deberían aclarar esta relación.

En resumen, a raíz de los resultados podemos concluir que la versión española de la Escala de Ensoñaciones Sexuales (Giambra y Singer, 1998) parece ajustarse mejor a una estructura trifactorial que a la propuesta unidimensional de sus autores para la versión anglosajona. Las tres subescalas han mostrado una adecuada consistencia interna y un patrón de correlaciones coherente con constructos afines. Esto implica la posibilidad de contar con un instrumento en nuestra lengua fiable, válido y útil para evaluar tres aspectos diferenciados de las 
ensoñaciones sexuales (tendencia a ensoñaciones heterosexuales, intensidad de las ensoñaciones sexuales y ensoñaciones eróticas personalizadas). No obstante, futuros estudios con diferentes muestras deberían ratificar estos primeros resultados obtenidos con la escala.

\section{REFERENCIAS BIBLIOGRÁFICAS}

Brislin, R.W. (1986). The wording and translation of research instruments. En W.J. Looner y J.W. Berry (Eds.), Fields methods in cross-cultural research (pp. 137-164). Newbury Park, CA: Sage.

Brown, J.J. y Hart, D.H. (1977). Correlates of female' sexual fantasies. Perceptual Motor and Skills, 45, 819-825.

Browne, M.W. y Cudeck, R. (1989). Single sample cross-validation indices for covariance structures. Multivaniate Behavioral Research, 24, 455-455.

Carmines, E.G. y Mclver, J.P. (1981). Analyzing models with unobservable variables. En GW. Bohrnstedt y E.F. Borgatta (Eds.), Social Measurement: Current Issues (pp. 65-115). Beverly Hills: Sage.

Carpintero, E. y Fuertes, A. (1994). Validación de la versión castellana del "Sexual Opinion Survey" (SOS). Cuadernos de Medicina Psicosomática, 31, 52-61.

Carretero-Dios, H. y Pérez, C. (en prensa). Normas para el desarrollo y revisión de estudios instrumentales. International Joumal of Clinical and Health Psychology, 5.

Carvajal, F., Sebastián, J., Comide, E., Delgado, A., Castellote, I. y Blanco, C. (1990). Implicaciones del modelo de la androginia en el comportamiento sexual: arousal subjetivo y fantasías sexuales. Revista de Psicología Social, 5, 23-42.

Desvarieux, A.R., Salamanca, Y., Ortega, V. y Sierra, J.C. (2005). Validación de la versión en castellano del Hurlbert Index of Sexual Fantasy: una medida de actitud hacia las fantasias sexuales. Manuscrito sometido a publicación.

Du Toint y Du Toint (2001). Interactive LISREL: User's Guide. Lincolnwood, IL: SSI.

Ellis, B.J. y Symons, D. (1990). Sex differences in sexual fantasy: An evolutionary psychological approach. Joumal of Sex Research, 27, 227-555. 
Fisher, W.A., Byrne, D., White, L.A. y Kelly, K. (1988). Erotophopiaerotophilia as a dimension of personality. Joumal of Sex Research, $25,123-151$.

García-Vega, E., Femández-García, P. y Rico Femández, R.A. (2005). Género y sexo como variables moduladoras del comportamiento sexual en jóvenes universitarios. Psicothema, 17, 49-56.

Giambra, L.M. (1977). Adult male daydreaming across the lifespam: A replication, further analysis, and tentative norms based upon retrospective reports. International Journal of Aging and Human Development, 8, 197-228.

Giambra, L.M. (1979). Sex differences in daydreaming and related mental activity from the late teens to the early nineties. Intemational Joumal of Aging and Human Development, 10, 1-34.

Giambra, L.M. (1983). Daydreaming in 40- to 60-year-old women: Menopause, health, values, and sexuality. Journal of Clinical Psychology, 39, 11-21.

Giambra, L.M. (2000). Frequency and intensity of daydreaming: Age changes and age differences from late adolescent to the old-old. Imagination, Cognition and Personality, 19, 229-267.

Giambra, L.M. y Martin, C.E.(1977). Sexual daydreams and quantitative aspects of sexual activity: Some relations for males across adulthood. Archives of Sexual Behavior, 6, 497-505.

Giambra, L.M y Singer, J.L. (1998). Sexual Daydreaming Scale of the Imaginal Process Inventory. En C.M. Davis, W.L. Yarber, R. Bauserman, G. Schreer y S.L. Davis (Eds.), Handbook of sexualityrelated measures (pp. 234-235). Londres: Sage.

Guttman, L. (1954). Some necessary conditions for common factor analysis. Psychometrika, 19, 149-161.

Hambleton, R.K. (1993). Translating achievement tests for use in cross-national studies. European Joumal of Psychological Assessment, 9, 54-65.

Hambleton, R.K. (1996). Adaptación de tests para su uso en diferentes idiomas o culturas: fuentes de error, posibles soluciones y directrices prácticas. En J. Muñiz (Ed.), Psicometría (pp. 207-238). Madrid: Universitas.

Heiman, J., LoPiccolo, L. y LoPiccolo, J. (1976). Becoming orgasmic: A sexual growth program for women. Englewood Cliffs, NJ: Prentice-Hall. 
Hicks, T. y Leitenberg, H. (2001). Sexual fantasies about one's partner someone else: Gender differences in incidence and trequency. Joumal of Sex Research, 38, 4-50.

Hsu, B., Kling, A., Kessler, Ch. y Knapke, K. (1994). Gender differences in sexual fantasy and behaviour in a college population: $A$ ten-year replication. Journal of Sex and Manital Therapy, 20, 103-118.

Hurlbert, D.F. y Apt, C. (1993). Female sexuality: A comparative study between women in homosexual and heterosexual relationships. Joumal of Sex and Marital Therapy, 19, 315-327.

Jöreskog, K.G (1970). A general method for analysis of covariance structures. Biometrika, 57, 239-251.

Kaiser, H.F. (1960). The application of electronic computers to factor analysis. Educational and Psychological Measurement, 20, 141-151.

Kaiser, H.F. (1970). A second generation Little Jiffy. Psychometrika, 35, 401-417.

Kaiser, H.F. (1974). An index of factorial simplicity. Psychometrika, 39, 31-36.

Kaplan, H.S. (1974). The new sex therapy. Nueva York: Brunner/Mazel.

Lameiras, M. y Failde, J.M. (1998). Sexualidad y salud en jóvenes universitarios /as: actitudes, actividad sexual y percepción de riesgo de la transmisión heterosexual del VIH. Análisis y Modificación de Conducta, 23, 27-63.

Leitenberg, H. y Henning, K. (1995). Sexual fantasy. Psychological Bulletin, 117, 469-496.

LoPiccolo, J. y Friedman, J.M. (1988). Broad spectrum treatment of low sexual desire: Integration of cognitive, behavioral, and systemic therapy. En S. R. Leiblum y R.C. Rosen (Eds.), Sexual desire disorders (pp. 107-144). Nueva York: Guilford Press.

LoPiccolo, J. y Steger, J. (1974). The Sexual Interaction Inventory: A new instrument for assessment of sexual dysfunction. Archives of Sexual Behavior, 2, 136-171.

Marsh, H.W., Balla, J.R. y Hau, K.T. (1996). An evaluation of incremental fit indices: A clarification of mathematical and empirical processes. En GA. Marcoulides y R.E. Schumacker (Ed.), Advanced structural equation modeling techniques (pp. 315-353). Hillsdale, NJ: Erlbaum.

McDonald, R.P. y Marsh, H.W. (1990). Choosing a multivariate model: Noncentrality and goodness-of-fit. Psychological Bulletin, 107, 247-255. 
Montero, I. y León, O.G (2005). Sistema de clasificación del método en los informes de investigación en Psicologia. International Journal of Clinical and Health Psychology, 5, 115-127.

Nutter, D. E. y Condron, M. K. (1983). Sexual fantasy and activity patterns of fernales with inhibited sexual desire versus normal controls. Journal of Sex and Marital Therapy, 9, 276-282.

Nutter, D. E. y Condron, M. K. (1985). Sexual fantasy and activity patterns of males with inhibited sexual desire and males with erectile dysfunction versus normal controls. Journal of Sex and Manital Therapy, 11, 91-98.

Ortega, V., Zubeidat, I. y Sierra, J.C. (2005). Further examination of the measurement properties of Spector, Carey, and Steinberg's Sexual Desire Inventory. Manuscrito sometido a publicación.

Purifoy, F.E., Grodsky, A. y Giambra, L.M. (1992). The relationship of sexual daydreaming to sexual activity, sexual drive, and sexual attitudes for women across the life-span. Archives of Sexual Behavior, 21, 369-385.

Renaud, C.A. y Byers, E.S. (1999). Exploring the frequency, diversity,


cognitions. Canadian Journal of Human Sexuality, 8, 17-30.

Renaud, C.A. y Byers, E.S. (2001). Positive and negative sexual cognitions: Subjective experience and relationships to sexual adjustment. Joumal of Sex Research, 38, 252-262.

Sierra, J.C. y Buela-Casal, G. (2004). Evaluación y tratamiento de las disfunciones sexuales. En G. Buela-Casal y J.C. Sierra (Eds.), Manual de evaluación y tratamientos psicologicos (pp. 439-485). Madrid: Pirámide.

Sierra, J.C., Ortega, V., Martín-Ortiz, J.D. y Vera-Villarroel, P. (2004). Propiedades psicométricas del Cuestionario de Wilson de Fantasías Sexuales. Revista Mexicana de Psicología, 21, 37-50.

Sierra, J.C., Ortega, V. y Zubeidat, I. (en prensa). Confirmatory factor analysis of a Spanish version of the Sex Fantasy Questionnaire: Assessing gender differences. Joumal of Sex and Marital Therapy.

Sierra, J.C., Vera-Villarroel, P. y Martín-Ortiz, J.D. (2002). Conductas sexuales, satisfacción sexual y fantasias sexuales: diferencias por género y nacionalidad. Avances en Psicologia Clínica Latinoamericana, $20,57-62$. 
Sierra, J.C., Zubeidat, I., Carretero-Dios, H. y Reina, S. (2003). Estudio psicométrico preliminar del Test del Deseo Sexual Inhibido en una muestra española no clínica. Revista Internacioal de Psicología Clínica y de la Salud/International Journal of Clinical and Health Psychology, 3, 489-504.

Singer, J.L. y Antrobus, J.S. (1963). A factor analytic study of daydreaming and conceptually related cognitive and personality variables. Perceptual and Motor Skills, 17 (supl. 3), 187-209.

Singer, J.L. y Antrobus, J.S. (1972). Daydreaming, imaginal process, and personality: A normative study. En P. Sheehan (Ed.), The function and nature of imagery (pp. 175-202). Nueva York: Academic Press.

Smith, D., Becker, L., Byrne, D. y Pryzbyla, D.P. (1993). Sexual attitudes of males and females as predictors of interpersonal attraction and marital compatibility. Journal of Applied Social Psychology, 23, 1001-1034.

Spector, I.P., Carey, M.P. y Steinberg, L. (1996). The Sexual Desire Inventory: Development, factor structure, and evidence of reliability. Joumal of Sex and Marital Therapy, 22, 175-190.

Steiger, J.H. (1990). Structural model evaluation and modification: An interval estimation approach. Multivariate Behavioral Research, 25, 173-180.

Tucker, L.R. y Lewis, C. (1973). The reliability coefficient for maximum likelihood factor analysis. Psychometrika, 38, 1-10.

Wilson, GD. (1978). The secrets of sexual fantasy. Londres: Dent.

Wilson, G.D. (1988). Measurement of sex fantasy. Sexual and Marital Therapy, 3, 45-55.

Wilson, G.D. (1997). Gender differences in sexual fantasy: An evolutionary analysis. Personality and Individual Differences, 22, 27-31.

Wilson, GD. y Lang, R. (1981). Sex differences in sexual fantasy patterns. Personality and Individual Differences, 2, 343-346.

Zeiss, A.M., Rosen, G.M. y Zeiss, R.A. (1977). Orgasm during intercourse: A treatment strategy for women. Joumal of Consulting and Clinical Psychology, 45, 891-895.

Zimmer, D., Borchardt, E. y Fischle, C. (1983). Sexual fantasies of sexually distressed and nondistressed men and women: An empirical comparison. Journal of Sex and Marital Therapy, 9, 38-50. 
Zubeidat, I., Ortega, V., Del Villar, C. y Sierra, J.C. (2003). Un estudio sobre la implicación de las actitudes y fantasías sexuales en el deseo sexual de los adolescentes. Cuadernos de Medicina Psicosomática y Psiquiatría de Enlace, 67/68, 71-78.

Zubeidat, I., Ortega, V. y Sierra, J.C. (2004). Evaluación de algunos factores determinantes del deseo sexual: estado emocional, actitudes sexuales y fantasías sexuales. Análisis y Modificación de Conducta, 30, 105-128.

Zubeidat, I. y Sierra J.C. (2003). Influencia de diferentes factores psicológicos sobre el deseo sexual. Revista Argentina de Clínica Psicológica, XII, 69-83.

Zurbriggen, E.L. y Post, M.R. (2004). Power, desire, and pleasure in sexual fantasies. Joumal of Sex Research, 41, 288-301. 


\section{ANEXO 1}

Versión traducida al español de la Sexual Daydreaming Scale (SDS) (Escala de Ensoñaciones Sexuales (Giambra y Singer, 1998).

1. Mis fantasías sobre el amor son vívidas, tengo la sensación $1 \begin{array}{llll}2 & 3 & 4 & 5\end{array}$ de que son reales (F2)

2. Yo me imagino fisicamente atractivo para las personas del $1 \begin{array}{lllll}1 & 2 & 3 & 4 & 5\end{array}$ sexo opuesto

3. Mientras estoy centrado en un trabajo, mi mente vaga en $\begin{array}{lllll}1 & 2 & 3 & 4 & 5\end{array}$ pensamientos sexuales (F1)

4. A veces en el trabajo me imagino haciendo el amor con una $\begin{array}{lllll}1 & 2 & 3 & 4 & 5\end{array}$ persona atractiva del sexo opuesto (F1)

5. Mis fantasías sexuales son muy vívidas y claras en mi $1 \begin{array}{llllll}1 & 2 & 3 & 4 & 5\end{array}$ mente (F2)

6. Mientras leo, frecuentemente me distraigo con fantasías $\begin{array}{lllll}1 & 2 & 3 & 4 & 5\end{array}$ sexuales (FI)

7. Mientras viajo en tren, autobús (o avión), mis pensamientos $\begin{array}{lllll}1 & 2 & 3 & 4 & 5\end{array}$ ociosos se vuelven amorosos (F3)

8. Cuando estoy aburrido, tengo fantasías sobre el sexo $1122 \quad 3 \quad 4 \quad 5$ opuesto (F1)

9. A veces a lo largo del día sueño con tener relaciones $\begin{array}{lllll}1 & 2 & 3 & 4 & 5\end{array}$ sexuales con alguien a quien aprecio (F3)

10. En mis fantasias tengo grandes deseos con alguien a quien $1 \begin{array}{llllll}1 & 2 & 3 & 4 & 5\end{array}$ admiro (F3)

11. Antes de dormirme, mis pensamientos ociosos se vuelven $\begin{array}{lllll}1 & 2 & 3 & 4 & 5\end{array}$ eróticos (F1)

12. Mis fantasías tienden a activarme fisicamente (F2)

\footnotetext{
1 Totalmente falso en $\mathrm{mi}$

2 Habirualmente falso en mi

3 Habitualmente cierto en mi

4 Cierto en mi

5 Mug clerto en mi
}

Nota: factor al que se asigna cada ítem entre paréntesis. 